\title{
Construction of a DNA vaccine based on the Mycobacterium tuberculosis Ag85A/MPT64 fusion gene and evaluation of its immunogenicity
}

\author{
HONG BAO, TING YU, YUFEN JIN, CHUNYAN TENG, XIMING LIU and YANLEI LI
}

The Second Hospital of Jilin University, Changchun 130041, P.R. China

Received April 14, 2012; Accepted September 7, 2012

DOI: $10.3892 / \mathrm{mmr} .2012 .1109$

\begin{abstract}
The aim of this study was to construct a DNA vaccine based on the Ag85A/MPT64 gene of Mycobacterium tuberculosis (MTB) and analyze its immunogenicity by enzyme-linked immunospot (ELISPOT) assay. The fusion gene encoding Ag85A/MPT64 was amplified by PCR from the genome of the MTB H37Rv strain and cloned into a eukaryotic expression vector followed by confirmation using restriction endonuclease digestion and DNA sequencing. The immunogenicity of the recombinant vector was tested in vivo in BALB/c mice. The serum antibody titers against Ag85A/MPT64 were detected by ELISPOT assay. The number of ELISPOT spots for the mice following immunization with Ag85A/MPT64 was significantly greater than for the negative and blank controls. A DNA vaccine based on the gene encoding the Ag85A/ MPT64 fusion protein of MTB was successfully constructed and expressed. Our data may serve as a foundation for further research into the prevention and treatment of tuberculosis and carcinomas.
\end{abstract}

\section{Introduction}

In recent years, with the emergence of drug-resistant or multidrug-resistant strains of Mycobacterium tuberculosis (MTB), the resurgence of tuberculosis has become a public health and social problem of worldwide concern. According to statistics released by the WHO in 2007, the global annual TB incidence is approximately 9.27 million cases and approximately 130 million people succumbed to the disease. The traditional BCG vaccine is very unstable and the protective immunity it provides to individuals from different parts of the world differ widely (1). Therefore, it is essential that studies

Correspondence to: Professor Ting Yu, The Second Hospital of Jilin University, No. 218 Zhiqiang Road, Changchun 130041, P.R. China

E-mail: yuting485@163.com

Key words: Mycobacterium tuberculosis, Ag85A, MPT64, DNA vaccine, ELISPOT concerning new tuberculosis vaccines and diagnostic reagents are carried out.

A new generation of DNA vaccines has been developed and has shown significant advantages in the prevention and treatment of a variety of infectious diseases and cancers (2). One prerequisite for the development of an effective vaccine is the selection of a highly immunogenic antigen. MTB culture filtrate proteins (CFPs) have been used as antigen compositions for TB prevention and in diagnostic reagents $(3,4)$. It has been found that the Ag85 complex is the major secreted protein antigen of MTB, accounting for $30 \%$ of the total secreted proteins (5). The antigen-specific response of $\mathrm{CD}^{+}$and $\mathrm{CD} 8^{+}$ $\mathrm{T}$ cells to an Ag85A DNA vaccine in BALB/c mice demonstrated that the Ag85A DNA vaccine was able to produce a strong and broad T-cell response and CTL activity (6). MTB secretes MTB protein 64 (MPT64), which is a major secreted protein in the early culture filtrate of MTB, accounting for $8 \%$ of total secreted proteins. The MPT64 protein has superoxide dismutase activity and a strong cellular immune activity with a specificity higher than that of tuberculin purified protein derivative (PPD) (7). In 1999, Kamath et al reported that the immunization of mice with MPT64 DNA vaccines induced spleen cells to produce IFN- $\gamma$ and also induced MPT64-specific CTL activity (8).

In order to combine the characteristics of the Ag85 and MPT64 proteins, a DNA vaccine was constructed and expressed in the present study. The recombinant proteins were purified and then validated by enzyme-linked immunospot (ELISPOT) assay.

\section{Materials and methods}

Materials. Pfu (Pyrococcus furiosus) Taq DNA polymerase, dNTPs, buffer and $\mathrm{MgCl}_{2}$ were purchased from Shanghai Sangon Biotech Co., Ltd. (Shanghai, China). The EcoRI and BamHI restriction enzymes, T4 DNA ligase kit and DNA markers were purchased from Takara Bio, Inc. (Shiga, Japan). The PCR product purification kit was purchased from Omega Bio-Tek, Inc. (Norcross, GA, USA). The gel extraction and plasmid extraction kits were purchased from Shanghai Huashun Bioengineering Co., Ltd. (Shanghai, China). The bacterial genomic DNA extraction kit was purchased from Shanghai Sangon Biotech Co., Ltd (Shanghai, China). The human IFN- $\gamma$ 
capture and IFN- $\gamma$ detection antibodies were purchased from R\&D Systems (Minneapolis, MN, USA). Phytohemagglutinin (PHA) was acquired from Sigma (St. Louis, MO, USA).

Animals. A total of 24 6-week-old special pathogen-free female C57BL/6 mice were purchased from the Experimental Animal Center of Jilin University, China. The mice were given free access to food and water throughout the study. The study was approved by the Institutional Animal Care and Use Committee of Jilin University, Changchun, China.

Genomic DNA extraction. Small amounts of MTB H37Rv were inoculated in $7 \mathrm{H} 9$ liquid medium and cultured at $37^{\circ} \mathrm{C}$ for two weeks. The liquid culture $(3 \mathrm{ml})$ was centrifuged at $12,300 \mathrm{rpm}$ for $1 \mathrm{~min}$. The pellet was resuspended in $50 \mu \mathrm{l}$ lysis buffer $(5 \mu 1$ 10X PCR buffer, $5 \mu 145 \mathrm{~g} / 1$ Tween-20, $5 \mu \mathrm{l}$ $45 \mathrm{~g} / 1 \mathrm{NP}-40,0.5 \mu 120 \mathrm{~g} / 1$ protease $\mathrm{K}$ and $34.5 \mu \mathrm{l}$ water) and kept at $55^{\circ} \mathrm{C}$ for $1 \mathrm{~h}$ and then in a boiling water bath for $10 \mathrm{~min}$ to inactivate proteinase $\mathrm{K}$. Following centrifugation at $12,300 \mathrm{rpm}$ for $1 \mathrm{~min}$, the supernatant was extracted twice with phenol/chloroform/isoamyl alcohol, followed by extraction twice with phenol/chloroform. The extract was precipitated using pre-cooled ethanol and then frozen at $-80^{\circ} \mathrm{C}$ for $10 \mathrm{~min}$. The precipitate was dissolved in $50 \mu \mathrm{l} \mathrm{TE}$ and stored at $-20^{\circ} \mathrm{C}$.

Primers. The Ag85A and MPT64 primers were synthesized by Invitrogen Life Technologies (Carlsbad, CA, USA). According to the gene coding sequences of MTB H37Rv Ag85A and MPT64, four primers were designed: P1, MPT64 forward primer 5'-GGAATTCCATGGTGCGCATCAAGATCTT-3' containing EcoRI restriction sites and start codon; P2, MPT64 reverse primer 5'-GGATCCCTAGGCCAGCATCGAGTC-3' containing BamHI sites and stop codon; P3, Ag85A reverse primer 5'-AGGATCCATGTTTTCCCGGCCGGGCTTG-3', containing Bam $\mathrm{HI}$ restriction sites and start codon; and $\mathrm{P} 4$, Ag85A reverse primer 5'-GGAATTCCTATGTTCGGAGCT AGGCGCCCTGGG-3', containing EcoRI restriction sites and stop codon. The PCR reaction system consisted of: $5 \mu \mathrm{l} 10 \mathrm{X}$ buffer, $1 \mu \mathrm{l}$ dNTPs $(2.5 \mathrm{mM}), 2 \mu 1 \mathrm{DNA}, 0.5 \mu \mathrm{l}(50 \mu \mathrm{M})$ primer and $0.5 \mu \mathrm{l}$ ( 2 units) Taq DNA polymerase, with water added to $50 \mu \mathrm{l}$. The reaction conditions were as follows: $95^{\circ} \mathrm{C}$ for $5 \mathrm{~min}$, $95^{\circ} \mathrm{C}$ for $45 \mathrm{sec}, 60^{\circ} \mathrm{C}$ for $30 \mathrm{sec}$ and $72^{\circ} \mathrm{C}$ for $60 \mathrm{sec}$, for 32 cycles, then $72^{\circ} \mathrm{C}$ for $10 \mathrm{~min}$. The PCR products were identified using $0.8 \%$ agarose gel electrophoresis.

Ag85A/MPT64 cloning plasmid construction. Doubledigested plasmid pcDNA3.1(+) and the PCR product were ligated by T4 DNA ligase at $22-24^{\circ} \mathrm{C}$ overnight and then transformed into DH5 $\alpha$ competent cells. The cells were cultured in 2YT medium containing $100 \mathrm{mg} / \mathrm{l}$ ampicillin. The blue/white method was used for screening positive clones. White colonies were selected randomly and inoculated into $4 \mathrm{ml}$ liquid $2 \mathrm{YT}$ medium containing ampicillin at $37^{\circ} \mathrm{C}$ with agitation overnight. Plasmid DNA was extracted using the alkaline lysis method. The plasmid DNA was digested with BamHI and EcoRI. The Ag85A (978 bp) and MPT64 (738 bp) fragments were detected using $0.8 \%$ agarose gel electrophoresis. Positive clones were sequenced by Shanghai Sangon Biotech Co.
Ag85A/MPT64 eukaryotic expression plasmid construction. Positive clones of the Ag85A/MPT64 recombinant plasmid were digested with $B a m \mathrm{HI}$ and $E c o \mathrm{RI}$ and the products were analyzed using $0.8 \%$ agarose gel electrophoresis. A PCR product of size 1716 bp was recovered using the PCR product purification kit and then dissolved in $35 \mu \mathrm{l}$ water and stored at $-40^{\circ} \mathrm{C}$. At the same time, an eukaryotic expression plasmid was prepared using the same method. Double-digested plasmid and DNA were ligated at $22-24^{\circ} \mathrm{C}$ overnight and then transformed into BL21 competent cells. The cells were cultured in 2YT medium containing $100 \mathrm{mg} / \mathrm{l}$ ampicillin. The blue/white method was used for screening positive clones. Positive clones were identified by BamHI and EcoRI double digestion.

DNA vaccine preparation and animal immunization. Following the mass culture of BL21 cells containing the Ag85A/MPT64 plasmid, the plasmid was extracted and then purified using phenol/chloroform/isoamyl alcohol (9). The concentration of plasmid was adjusted to $1.5 \mathrm{~g} / 1$ with sterile saline. Eight $\mathrm{BALB} / \mathrm{c}$ mice were injected with $60 \mu \mathrm{l} 8.5 \mathrm{~g} / \mathrm{l}$ pivacaine in the side of the thigh. Three weeks after pretreatment, the mice were immunized once with $50 \mu \mathrm{g}$ Ag85A/MPT64 plasmid. The vector was injected intramuscularly in a $60 \mu \mathrm{l}$ volume. The control mice were immunized using the blank plasmid and sterile saline, respectively ( 8 mice per group).

ELISPOT. Anticoagulant (3-4 ml) was taken from the mouse tail vein, and after centrifugation, the lymphocyte layer was separated from mouse serum, followed by purification of blood mononuclear cells and adjustment of the cell concentration. The positive control (PHA), negative control (normal saline), blank control (plasmid) and different dilutions of the cell suspension were added to a pre-packed IFN- $\gamma$ capture monoclonal antibody microplate. The cells were cultured at $37^{\circ} \mathrm{C}$ in $5 \% \mathrm{CO}_{2}$ overnight. The supernatant was discarded and the plates were washed with PBST. The detection antibody was added to each well and the plate was incubated at $37^{\circ} \mathrm{C}$ for $2 \mathrm{~h}$. After washing, the substrate solution was added to each well and the plate was incubated for a further $30 \mathrm{~min}$. The plates were washed, the reaction was terminated and the results were observed.

\section{Results}

Ag85A/MPT64 gene cloning. A specific band was amplified from a template of MTB H37Rv genomic DNA by PCR. Following double digestion, the PCR products were cloned into the plasmid and then transformed into DH5a competent cells. White colonies were selected randomly and the plasmids were extracted and double-digested. A 978-bp fragment and a 738-bp fragment were obtained (Fig. 1A). Bi-directional sequencing results were consistent with those previously reported (10).

Ag85A/MPT64 vector construction. Plasmid DNA was extracted after positive clones were selected and cultured. Following double enzyme digestion, a 1716-bp fragment was recovered and then ligated with the plasmid fragment that was digested using the same enzyme. Positive clones in the transformed plates were selected randomly. A 1716-bp fragment 

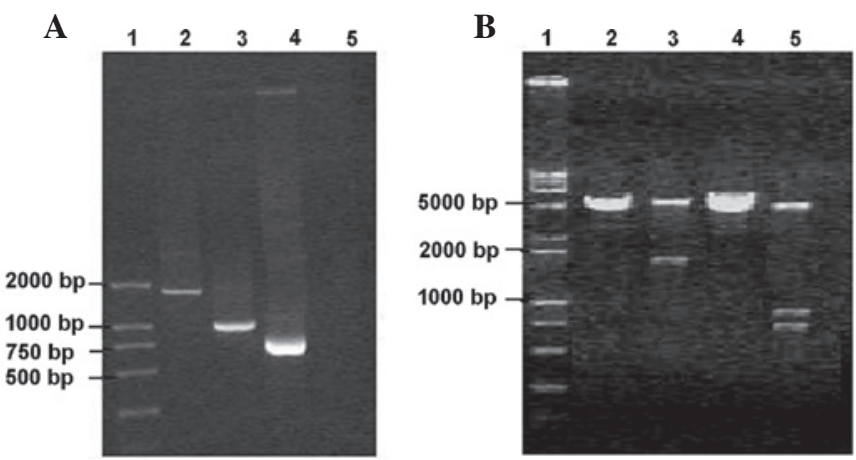

Figure 1. Detection of PCR products and vector restriction sites using agarose gel electrophoresis. (A) Electrophoresis results of the PCR products. Lane 1, DNA marker; 2, PCR fragment of Ag85A and MPT64 fusion gene; 3, PCR product of Ag85A gene; 4, PCR product of MPT64 gene; and 5, negative control. (B) Electrophoresis results for vector digestion products. Lane 1, DNA marker; 2, product of plasmid pcDNA3.1 (+) by single EcoRI digestion; 3 , product of positive clone by single EcoRI digestion; 4, product of plasmid pcDNA3.1 (+) by double digestion; and 5, product of positive clone by double digestion.

was obtained after the plasmid was digested with EcoRI and 2978-bp and 738-bp fragments were obtained after the plasmid was double-digested (Fig. 1B). The nucleotide sequence of the recombinant gene encoding Ag85A/MPT64 had no mutations, and the gene was correctly inserted into the vector.

ELISPOT. As shown in Fig. 2, the numbers of ELISPOT spots for the mice immunized with Ag85A/MPT64 were significantly greater than for the negative and blank controls. The vaccine-stimulated production of immunoglobulin (Ig) and cytokines may be screened effectively by ELISPOT assay (11), which is important for vaccine research. The soluble protein in the cells forms clearly visible spots through a color reaction and these spots may be directly and manually counted under a microscope. The spots may also be counted by an ELISPOT analysis system and screened by high-throughput screening, which is far more efficient than other detection methods.

\section{Discussion}

A 4,400-kb fragment of MTB contains more than 4,000 genes and these genes encode proteins that may cause strong cellular and humoral immune responses. Therefore, these genes, including HSP, MPT64, Ag85, Esat-6 and PstS-3, may be considered as candidate genes for DNA vaccines (12).

The Ag85 complex includes three subunits, Ag85A, $\mathrm{Ag} 85 \mathrm{~B}$ and $\mathrm{Ag} 85 \mathrm{C}$, with relative molecular weights of $30 \times 10^{3}-32 \times 10^{3} \mathrm{kDa}$, which exist in the MTB and BCG cell walls and culture filtrates. The Ag85 content is highest in the MTB cell wall proteins and secreted proteins and comprises $31-45 \%$ of the total protein (13). Ag85A and PstS-3 DNA vaccines have been used to treat $\mathrm{C} 57 \mathrm{BL} / 6 \mathrm{~J}$ mouse tuberculosis infection and it was found that DNA vaccines can prevent the recurrence of latent tuberculosis infection in mice and shorten the cycle of conventional chemotherapy. Significant reductions in the load of MTB in the lung and spleen occurred when a Ag85A DNA vaccine was used alone or in combination with chemotherapy to treat multidrug-resistant tuberculosis in

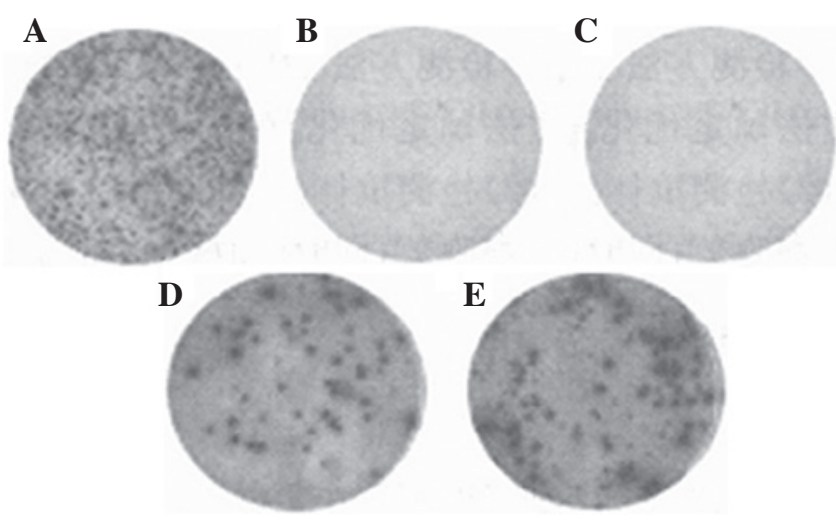

Figure 2. ELISPOT detection of the color reaction of DNA vaccine. (A) positive control; (B) saline negative control; (C) plasmid blank control; (D and E) experimental group with different dilutions of the serum. ELISPOT, enzyme-linked immunospot.

mice for 2 months (14). MPT64 is a major secretory protein of the MTB complex group and is also a key T-cell antigen. MPT64 and ESAT6 DNA vaccines were used to immunize mice and, by observing the pathological changes in the lungs of the MTB-infected mice 4 weeks after abdominal challenge to evaluate the protective strength of the DNA vaccine, it was found that the changes in the MPT64 and ESAT6 DNA vaccine groups were similar to those in the BCG group. This is because most of the mice experienced the proliferative response and a small number of the mice experienced a tissue reaction.

Ag85 is abundant in the cell wall and culture filtrates of MTB and BCG cells. The mycolic acid transferase activity of Ag85 has an important role in the later stages of the synthesis of the MTB cell wall. Ag85A, Ag85B and Ag85C have highly homologous sequences (13). The Ag85/MPT64 protein is capable of stimulating the body to produce a large amount of IFN- $\gamma$ and induces strong T-cell proliferation. Mice were able to resist MTB attack following immunization with Ag85/MPT64 (15). It has also been reported that high levels of Ag85/MPT64 antibodies were detected in the serum following the infection of individuals with TB bacilli (16).

Antigens are a feasible alternative to BCG. When BCG enters the bladder, it combines with the tumor cell surface fibronectin (FN) or bladder epithelial cells and causes an immune response. This combination confers the characteristics of the Ag85 complex, known as the fibronectin-binding antigen $(12,13)$. The Ag85 protein was expressed in some regions of the bladder following the infusion of a eukaryotic expression vector into the bladder. Therefore, it appears that the bladder wall absorbs the eukaryotic expression vector DNA vaccine.

An Ag85A/MPT64 DNA vaccine has been successfully constructed in this study and it was confirmed that specific antibodies against MTB were produced following animal immune challenge.

ELISPOT assays are more specific and sensitive than TST, which is helpful for the screening and monitoring tuberculosis infection of patients undergoing anti-tumor necrosis factor (TNF) treatment (17). A IFN- $\gamma$ test using TB-specific antigens enables the efficient diagnosis of latent TB infection (LTBI) in HIV-infected individuals. ELISPOT assays with their 
high sensitivity and specificity play an important role in TB screening. Due to the high incidence of tuberculosis and BCG vaccination in China, investigations may be carried out using this technology among tuberculosis-exposed populations to exclude the effect of TST and PPD false positives and false negatives and for the early diagnosis of tuberculosis-infected individuals According the degree of reaction, preventive treatment may be carried out if necessary, which will reduce the incidence and the source of infection.

\section{References}

1. Kaufmann SH: Is the development of a new tuberculosis vaccine possible? Nat Med 6: 955-960, 2000.

2. Fuller DH, Rajakumar PA, Wilson LA, et al: Introduction of mucosal protection against primary, heterologous simian immunodeficiency virus by a DNA vaccine. J Virol 76: 3309-3317, 2002.

3. Havlir DV, Wallis RS, Boom WH, Daniel TM, Chervenak K and Ellner JJ: Human immune response to Mycobacterium tuberculosis antigens. Infect Immun 59: 665-670, 1991.

4. Weldingh K, Rosenkrands I, Jacobsen S, Rasmussen PB, Elhay MJ and Andersen P: Two-dimensional electrophoresis for analysis of Mycobacterium tuberculosis culture filtrate and purification and characterization of six novel proteins. Infect Immun 66: 3492-3500, 1998.

5. Malin AS, Huygen K, Content J, et al: Vaccinia expression of Mycobacterium tuberculosis-secreted proteins: tissue plasminogen activator signal sequence enhances expression and immunogenicity of M. tuberculosis Ag85. Microbes Infect 2: $1677-1685,2000$.

6. Romano M, Roupie V, Wang XM, et al: Immunogenicity and protective efficacy of tuberculosis DNA vaccines combining mycolyl-transferase Ag85A and phosphate transport receptor PstS-3. Immunology 118: 321-332, 2006.

7. Oettinger T, Holm A and Hasløv K: Characterization of the delayed type hypersensitivity-inducing epitope of MPT64 from Mycobacterium tuberculosis. Scand J Immunol 45: 499-503, 1997.
8. Kamath AT, Feng CG, Macdonald M, Briscoe $\mathrm{H}$ and Britton WJ: Differential protective efficacy of DNA vaccines expressing secreted proteins of Mycobacterium tuberculosis. Infect Immun 67: 1702-1707, 1999

9. Tehranchian S, Akbarzadeh T, Fazeli MR, Jamalifar H and Shafiee A: Synthesis and antibacterial activity of 1-[1,2,4-triazol-3-yl] and 1-[1,3,4-thiadiazol-2-yl]-3-methylthio6,7-dihydrobenzo[c]thiophen-4(5h)ones. Bioorg Med Chem Lett 15: 1023-1025, 2005.

10. Wang Z, Potter BM, Gray AM, Sacksteder KA, Geisbrecht BV and Laity JH: The solution structure of antigen MPT64 from Mycobacterium tuberculosis defines a new family of beta-grasp proteins. J Mol Biol 366: 375-381, 2007.

11. Bastos RG, Ueti MW, Knowles DP and Scoles GA: The Rhipicephalus (Boophilus) microplus Bm86 gene plays a critical role in the fitness of ticks fed on cattle during acute Babesia bovis infection. Parasit Vectors 3: 111, 2010.

12. Choi SS, Chung E and Jung YJ: Newly identified CpG ODNS, M5-30 and M6-395, stimulate mouse immune cells to secrete TNF-alpha and enhance Th1-mediated immunity. J Microbiol 48: 512-517, 2010.

13. de Klerk LM, Michel AL, Bengis RG, Kriek NP and Godfroid J: BCG vaccination failed to protect yearling African buffaloes (Syncerus caffer) against experimental intratonsilar challenge with Mycobacterium bovis. Vet Immunol Immunopathol 137: 84-92, 2010.

14. Liang Y, Wu X, Zhang J, et al: The treatment of mice infected with multi-drug-resistant Mycobacterium tuberculosis using DNA vaccines or in combination with rifampin. Vaccine 26 : 4536-4540, 2008.

15. Ingolotti M, Kawalekar O, Shedlock DJ, Muthumani K and Weiner DB: DNA vaccines for targeting bacterial infections. Expert Rev Vaccines 9: 747-763, 2010.

16. Cai H, Tian X, Hu XD, Zhuang YH and Zhu YX: Combined DNA vaccines formulated in DDA enhance protective immunity against tuberculosis. DNA Cell Biol 23: 450-456, 2004.

17. Takeno M, Murakami S and Ishigatsubo Y: Tuberculosis associated with anti-TNF therapy. Nippon Rinsho 65: 1308-1313, 2007 (In Japanese). 